\title{
A MULTI-LEVEL SCALE OF TECHNICAL SAFETY INDICATORS OF URBAN LIFE SUPPORT SYSTEMS
}

\author{
Sergei Gennadievich Emelianov ${ }^{1 *}$ Natalya Vladimirovna Bakaeva ${ }^{1}$ Vladimir Alexandrovich Gordon ${ }^{2}$ \\ ${ }^{1}$ Southwest State University, Kursk, Russia \\ ${ }^{2}$ Orel State University, Orel, Russia
}

The article describes a multilevel scale of quantitative indicators of technical safety of an urban life support system. The described system is considered as a territorially - distributed structure with a complex of interacting subsystems. Indicators are presented in the form of multi-component vectors and describe conformity of the actual state of the system to regulatory requirements. As an example, Indicators of technical safety of an urban water supply subsystem are calculated.

Key words: Technical safety, Urban life support systems, Urban water supply

\section{INTRODUCTION}

Most individual and social people's needs are satisfied by a town, a multi-level natural and social-economic territorial system within which inter related processes of external and internal population life support take place.

Modern towns have a complex infrastructure that provides conditions for population house hold activities, work, and leisure. An urban life support

system consists of a number of interacting sub systems performing functions subject to certain requirement sand interaction conditions. Thus, an urban life support system can be refer red to as a multi-purpose territorially-distributed structure (TDS) [01,02].

Damage to any subsystem as a result of external impact or failure can cause malfunction of the whole life support system and lead to undesirable consequences for population. Forecasting life support system damage results is an important task when planning activities to ensure urban environment safety.

«When solving safety problems, a territorially-distributed structure is considered as a uniform formation united by a common border either according to administrative division or on the basis of functional purposes. Generally, it includes a number of constituents, as a rule, varying in characteristics, purposes, significance"[01].

\section{STATEMENT OF THE RESEARCH PROBLEM}

One of the objectives of urbanized area integrated safety ensuring is the establishment of balance of relations between the standard-compliant and actual state of the life support system of urbanized areas and recommendations for a gradual step wise reduction of leading to hazards and risks imbalance.

As a preliminary stage of the creation of a TDS's safety hazard model, assessment of structure's safety state including TDS's constituents is required. Development of a quantitative indicators list reflecting the current state of the system is an important basis for the assessment of system's safety level. It is impossible to proceed with scientific management without information a bout the system state presented in the form of certain qualitative and quantitative indicators. It is proposed to perform TDS's state assessment by means of a dimension less integrated indicator that characterizes compliance of the actual urban are a life support system state with regulatory requirements calculated according to structure monitoring (audit) data using expert methods.

To assess the urban life support system state and system constituents at the preliminary stage formation of initial data on the basis of expert information and monitoring takes place. Initial data include:

- life support subsystem infrastructure diagrams;

- life support subsystem process flow diagrams;

- subsystems interaction diagrams;

- a table of subsystem elements weights and weights of subsystems comprising the system;

- life support subsystem operational parameters and modes;

- operation process characteristics and their dependence on the elements state.

The state of a life support subsystem is proposed to be assessed by means of a dimension less integrated indicator that is calculated according to the following procedure:

1. Analysis of the implementation of legal and regulatory requirements, legal acts and technical regulations and conditions related to functioning issues. Analysis of technical and design project documentation in order to identify elements (sections, areas, facilities, instruments, equipment etc.) of hazard of failure, destruction and etc.

2. Audit of structure's design and space-planning solutions. For example, for fire hazard assessment, admissible areas of fire compartments are identified, conformity of fire resistance limits for structures, requirements for 
escape routes are verified, an unobstructed access for fire-fighters or access roads for fire vehicles to the site of fire are checked.

3. Audit of technical means and systems providing life support subsystem functions (check of availability, state and use). For example, for fire hazard assessment they are external fire fighting, internal fire fighting water supply, lightning protection, an automatic fire alarm system, a smoke removal system, an automatic fire-fighting system, public address and general alarm and evacuation control systems, primary fire-fighting means.

4. Audit of structure's engineering systems: ventilation, power supply, gas supply, water supply, heat supply, lifts, in terms of this subsystem functioning support (e.g.in terms of ensuring object's fire safety).

5. Audit of activities connected with personnel (population) safety (evacuation, rescue) in case of accident sand emergencies including administrative and technical measures: management and personnel competence in the field of emergencies handling, availability of required organizational documents and plans for prevention and response to emergencies, escape plans and routes.

\section{RESULTS}

Let us consider an urban water supply subsystem "Water and Wastewater Treatment Plant" (Figure 1) as an example.

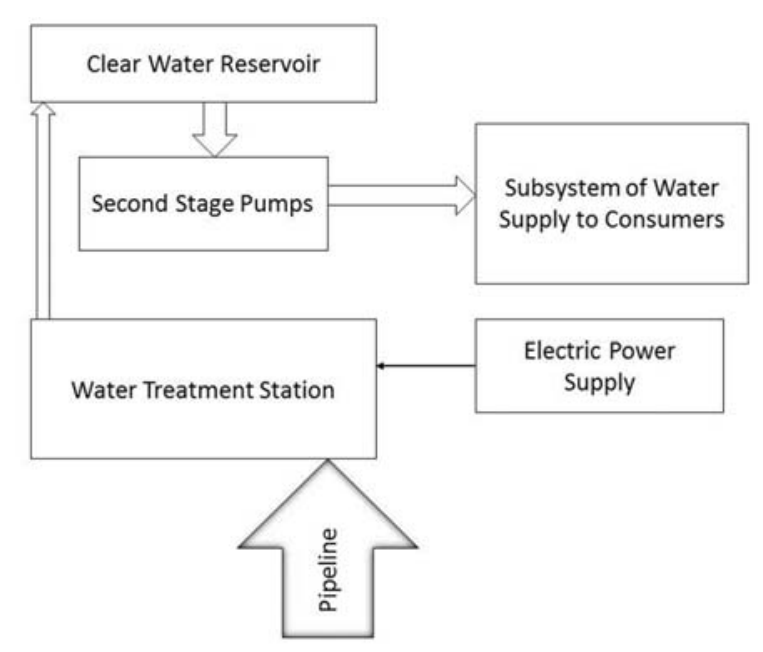

Figure 1: Water supply subsystem "Water and Wastewater Treatment Plant"

This subsystem is the most important part of the life support system for population in the place of living, and consists of six interacting subsystems connected by means of communication (Figure 2).

Water supply subsystem "Water and Wastewater Treatment Plant" is presented in the form of a directed graph, whose nodes correspond to the blocks of infrastructure elements, and edges - to the ties between blocks (Figure 3).

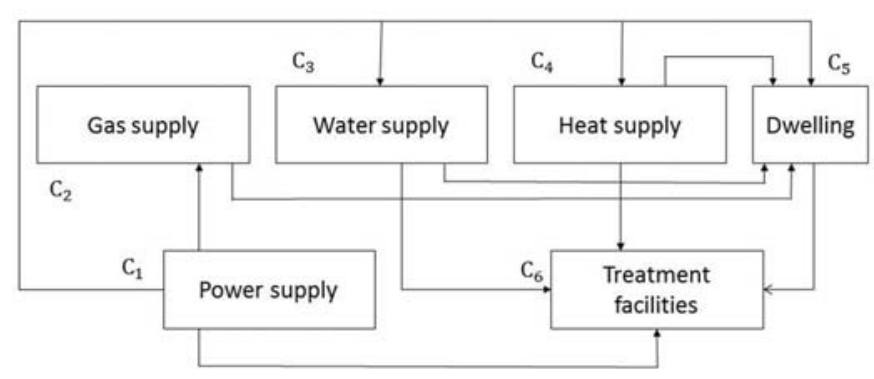

Figure 2: Diagram of urban life support systems interaction by means of communication

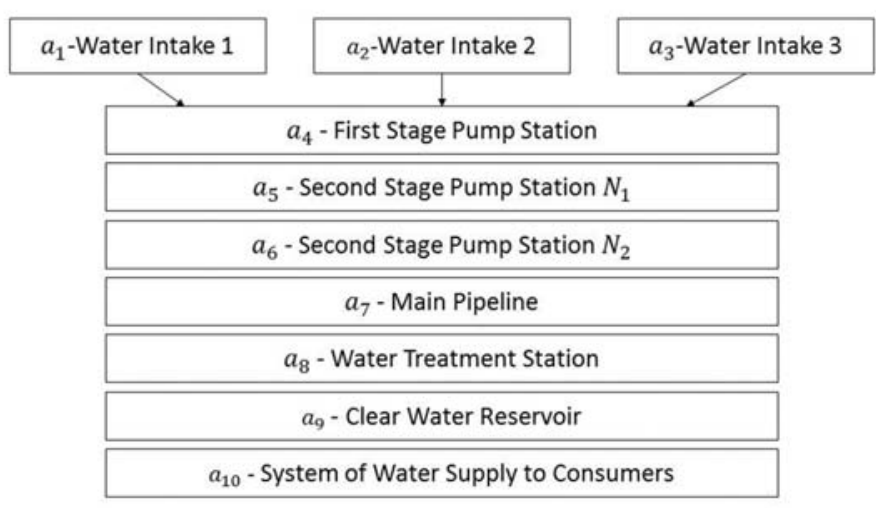

Figure 3: Diagram of water supply subsystem in the form of a directed graph

In this way a water supply subsystem is studied in [03] where subsystem's state forecasting in case of emergency caused by a tsunami is studied. Figures 1, 2, 3 and the follow in description are borrowed from this work. "The directed edge in the graph going from one node to another one means that damage in the first node (infrastructure element block) causes damage to the element block corresponding to the second node. The task of the experts in the process of "infrastructure diagram" building is to arrange elements of life support system infrastructure in blocks in such a way that the resulting infrastructure diagram presents the infrastructure in the form of large blocks" (Figure 3).

In the diagram of the life support subsystem infrastructure "Water and Water Treatment Plant" there are links $\left(a_{1}, a_{4}\right), \quad\left(a_{2}, a_{4}\right),\left(a_{3}, a_{4}\right),\left(a_{8}, a_{9}\right)$ between the nodes. It means that damage to the infrastructure element "Water Treatment Station" can cause damages to the element "Clear Water Reservoir" due to some design features of the subsystem under study: the clear water reservoir is located inside the water treatment station. Similarly, due to the design features of the water intake, the first-stage pump station can be damaged as well. There st elements of "Water and Water Treatment Plant" infrastructure are independent, i.e. their damage does not cause damage of other elements.

Using the algorithm, proposed in [04-06], let us conceive the life support subsystem "Water and Water Treatment Plant" as a combination of groups consisting of $j_{k}$ uniform structures. Each combination $\mathrm{n}$ has to meet $i_{k}$ 
regulatory requirements according to which its normal operation is provided. Let us denote standard value of the requirements for the element as $a_{k i}^{\mathrm{H}}$, where $k$ is the number of the element group, $i$ is the number of the requirement for the element of this group (Figure 4).

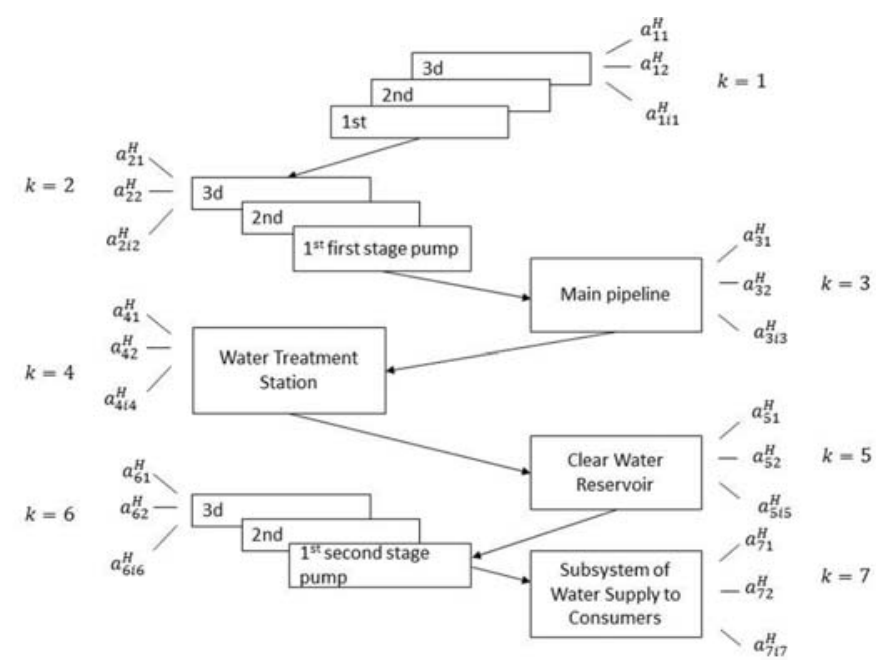

Figure 4: Diagram of regulatory requirements for water supply subsystem

Thus, this subsystem consists of 7 groups of uniform elements, i.e. $k=1, \ldots 7$. Andthefirstgroup "Water Supply Intake" consists of $j_{1}$ elements, as, for example, in the diagram in Figure $4 j_{1}=1,2,3$.

These con d group "First stage pump" consists of $j_{2}$ elements, $j_{2}=1,2,3$ etc.

The third, fourth and fifth groups are independently operating elements, i.e. $j_{3}=j_{4}=j_{5}=j_{7}=1$.

The sixth group "First stage pump" consists of $j_{6}$ elements, $j_{6}=1,2,3$.

Regulatory requirements for each element are presented in the form of a multi-component vector $\bar{A}_{k}^{H}(k=1,2, \ldots 7)$

$$
\bar{A}_{k}^{H}=\left\{a_{k_{1}}^{H}, a_{k_{2}}^{H} \ldots a_{k i_{k}}^{H}\right\}
$$

Experts assign to each requirement $a_{k_{i}}^{\mathrm{H}}$ value $l_{k i}$ ,a weighing factor, characterizing the level of this requirement significance $\left(0 \leq l_{k i} \leq 1\right)$. The sum-total of weighing factors is subject to condition $\sum_{i=1}^{i k} l_{k i}=1$. Weighing factors $l_{k i}$, corresponding to each vector component $\bar{A}_{k}$ are also represented by vectors components $\bar{L}_{k}=\left\{l_{k_{1}} l_{k_{2}} \ldots l_{k i_{k}}\right\}$.

As are result of the performed audit, actual values of group element parameters comprising life support subsystem "Water and Wastewater Treatment Plant" are determined. By means of comparison $a_{k i j}^{\phi}$ of actual values of $i$-th requirement implementation and requirement for $j$-th element of $k$-th qroup with regulatory standard $a_{k i}^{\mathrm{H}}$, their ratios $\lambda_{k i j}=\frac{a_{k i j}^{\phi}}{a_{k i}^{\mathrm{H}}}$, or $\frac{a_{k i}^{\mathrm{H}}}{a_{k i j}^{\phi}}$ are calculated showing the degree of the nearness of the actual value of the parameter o the standard value.

Characteristics $\lambda_{k i j}$ changes from 0 (complete nonconformity of the actual parameter to the regulatory require- ment) to 1 (complete conformity). Calculating fact or $\mathrm{s}$ the following shall be considered:

- if the regulatory standard value is given in the form of in equality, then characteristics $\lambda_{k i}$ is taken equal to 1 , when the actual parameter value satisfies this inequality;

- if the standard value of the parameter is a fixed value, then characteristics $\lambda_{k i}$ is taken equal to $1(0)$, when the actual value is equal to this value (is not equal);

- in some cases when the numerical actual value of the parameter differs from the regulatory standard one, characteristics $\lambda_{k i}$ value should be taken equal to zero;

- if the regulatory requirement for the structure is qualitative (yes/no), then characteristics $\lambda_{k i}$ equalsto1(0), when the regulatory and actual requirements are equal (opposite) in meaning.

Similar to vector $\bar{A}_{k}^{H}$, let us introduce vectors $\bar{\lambda}_{k j}$, which components are the characteristics: $\lambda_{k i j}: \bar{\lambda}_{k j}=\left\{\lambda_{k_{1} j} \lambda_{k_{2} j} \ldots \lambda_{k i_{k} j}\right\}$

Let us determine the indicator of the element state of life support subsystem $\eta_{k j}$ as a scalar product of vectors $\bar{\lambda}_{k}$ and $\bar{L}_{k}$

$$
\eta_{k j}=\bar{\lambda}_{k j} \bar{L}_{k}=\lambda_{k_{1} j} l_{k_{1}}+\lambda_{k_{2} j} l_{k_{2}}+\cdots \lambda_{k i_{k} j} l_{k i_{k}}
$$

Then, if a group consists of $j_{k}$ uniform elements which state is estimated by indicators $\eta_{k j}$, the indicator of the state of the whole group $\eta_{k}$ is determined as an arithmetic average of the indicators of the elements of the group:

$$
\eta_{k}=\frac{\eta_{k_{1}}+\eta_{k_{2}}+\ldots \eta_{k j_{k}}}{j_{k}}
$$

Assessing the weighting of the group of elements of the subsystem with vector $\bar{L}_{\Pi}=\left\{l_{1} l_{2} \ldots l_{k}\right\}$, which components satisfy the condition $\sum_{n=1}^{k} l_{n}=1$, we obtain the indicator of this subsystem state $\eta_{\Pi}$, which is determined by the scalar product of vectors of parameters of groups $\bar{V}_{\Pi}=\left\{\eta_{1} \eta_{2} \ldots \eta_{k}\right\}$ and the weighting vector of the group $\bar{L}_{\Pi}$.

$$
\eta_{\Pi}=\bar{V}_{\Pi} \bar{L}_{\Pi}=\eta_{1} l_{1}+\eta_{2} l_{2}+\cdots \eta_{k} l_{k}
$$

Indicators of the state of other life support subsystems are calculated in a similar way.

Introducing a subsystem weighting vector $\bar{L}=\left\{l_{\Pi_{1}} l_{\Pi_{2}} \ldots l_{\Pi_{t}}\right\}$ which components satisfy the condition $\sum_{r=1}^{t} l_{\Pi r}=1$ where $l_{\Pi r}(r=1,2, \ldots t)$ is the weighting of $r$-th subsystem in the life support system of the area, $t$ is the number of subsystems, let us determine the area (region, town, district, etc.) life support system state as a scalar product of vectors $-\bar{L}$ and $\bar{\eta}=\left\{\eta_{\Pi_{1}} \eta_{\Pi_{2}} \ldots \eta_{\Pi_{t}}\right\}$ :

$$
\eta_{\mathrm{T}}=\bar{L} \bar{\eta}=\eta_{\Pi_{1}} l_{\Pi_{1}}+\eta_{\Pi_{2}} l_{\Pi_{2}}+\cdots+\eta_{\Pi_{t}} l_{\Pi_{t}}
$$




\section{CONCLUSION}

Thus, a multi-level scale of indicators of an urban life support system state including characteristics of subsystem elements, subsystems themselves, territorial total of subsystems etc. was built. Due to the structure of indicators both territorial and department al comparisons can be made and system state changes can be compared.

The proposed indicators and methods of their calculation can facilitate the development of the quailmetry of safety levels of different kinds of activities in urban areas and optimization of urban life support system safety management.

\section{REFERENCES}

1. Kalinin A. A. Visualization of functioning of territorially-distributed structures // Kalinin A.A. Applied Informatics. -2006. - N6. - pp.11-18.

2. Korolev V. I. Methods for territorially-distributed structure safety hazards model creation/Korolev V.I., Novikov A.A., Kuzmin A.P., Shornikov A.N.//Online magazine "Techno sphere safety technology" 2013. - Issue 2(48). - pp.1-12.

3. Andreev A.K. Expert forecasting of the effects of life support system damages in case of emergencies/ Andreev A.K, Kamaev D.A., Trakhtengerts E.A.// Large systems management - 2009. - Issue 25.-pp. 241-293.

4. Bruma E.V.A multi-level scale of indicators of urban areas fire safety/BrumaE.V.// Biosphere compatibility: people, regions, technologies-2013.-N3.-pp.3-11.

5. Kolchunov V.I.Method for calculation of the indicator of public buildings and facilities accessibility for people with limited mobility// Kolchunov V.I.,Skobeleva E.A.,Bruma E.V.//BuildingandReconstruction.-2013, №4(48).-pp.60-68.

6. Gordon V.A. Assessment of the availability and ecological safety of urban environment//Gordon V.A., Pilipenko O.V., Bruma E.V.//LAPLambertAcademicPublishing.-2016.-148p. 